\title{
Through-Container Measurement of Acoustic Signatures for Classification/Discrimination of Liquid Explosives (LEs) and Precursor Threat Liquids
}

\author{
Aaron A. Diaz, Todd J. Samuel, Brian J. Tucker, Anthony D. Cinson \\ Juan D. Valencia, Kevin L. Gervais, Jason S. Thompson \\ Pacific Northwest National Laboratory, 902 Battelle Blvd, Richland, WA., 99352
}

\begin{abstract}
Work at the Pacific Northwest National Laboratory has demonstrated that ultrasonic property measurements can be effectively employed for the rapid and accurate classification/discrimination of liquids in small, carryon, standard "stream-of-commerce" containers. This paper focuses on a set of laboratory measurements acquired with the PNNL prototype device as applied to several types of liquids (including threat liquids and precursor chemicals) to the manufacture of LEs in small commercially available plastic containers.
\end{abstract}

Keywords: acoustic, ultrasonic, acoustic velocity, attenuation, noninvasive, database

\section{INTRODUCTION}

Emerging homeland security threats and increasingly sophisticated adversaries have heightened the need for effective technologies that can classify, sort and discriminate the contents of small and large containers, from liquids to bulk solids, within a few seconds. Current technologies are limited by prolonged laboratory analysis, high false positive readings and extensive training required for operators.

An advanced technology developed at Pacific Northwest National Laboratory in Richland, WA, addresses those challenges using a patended acoustic measurement technology. PNNL has developed several different acoustic based inspection devices that leverage the decades of expertise in applied ultrasonic physics, electronics, and custom software development that PNNL possesses.

The PNNL acoustic based inspection technology can be used to inspect, classify, sort and discriminate the contents of sealed containers, while doing so in a way that is non-intrusive and non-invasive to the containers being screened. PNNL's acoustic technology platforms have evolved over the past 15 years to become essential tools employed by government agencies domestically and internationally in detecting and interdicting smuggled and dangerous materials at borders.

The unique characteristics of PNNL acoustic-based inspection methodologies allows them to be used to perform the following primary functions:

- $\quad$ Detect contraband and hidden compartments in liquid-filled containers and solid form commodities,

- $\quad$ Sort liquid types into groups of like and unlike

- $\quad$ Classify liquids and solids as a function of temperature

- $\quad$ Determine the fill-level in liquid-filled containers, and

- $\quad$ Rapidly acquire data, providing easy-to-interpret measurement results 
The latest evolution of this technology is called the Container Screening Device (CSD). It is a bench-top system used for real-time, sealed-container contents inspection and classification (Figure 1). The CSD was designed to inspect and classify/discriminate the liquid contents in smaller containers such as shampoo bottles, soda cans, glass containers and other containers as large as 10-inches in diameter using precharacterized acoustic signatures in its tablet personal computer (PC) database.

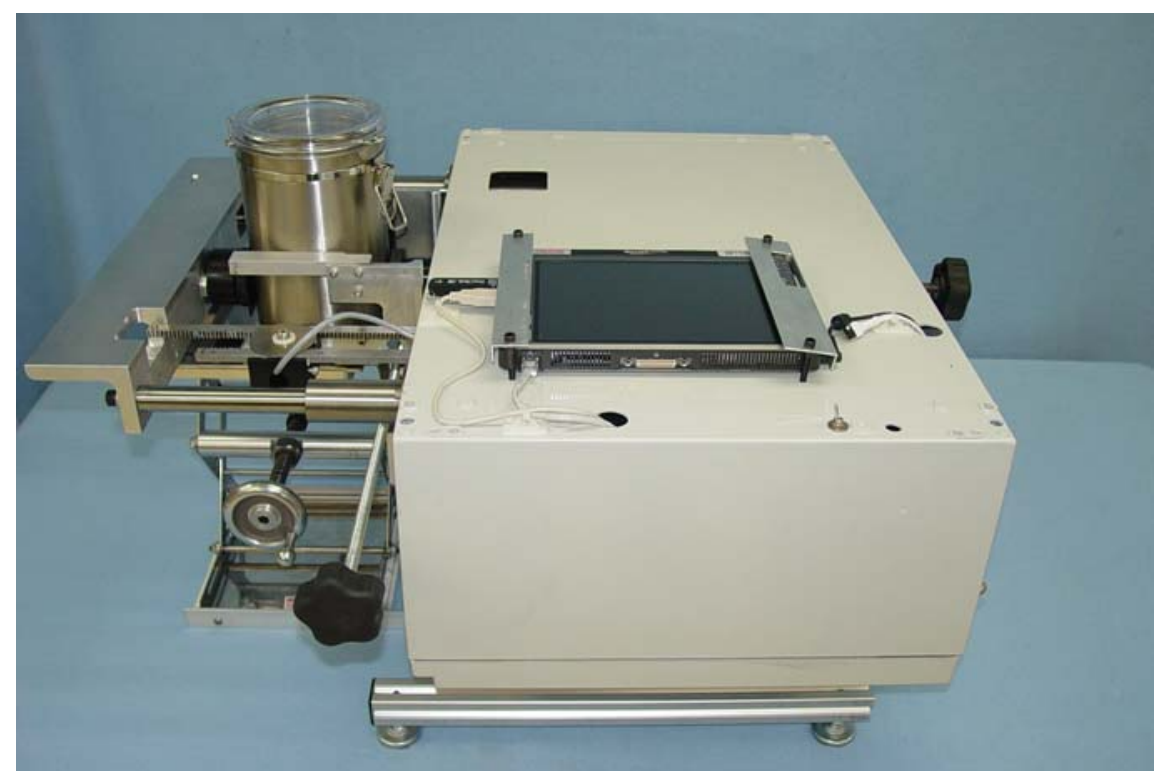

Figure 1. The Container Screening Device (CSD) Alpha Unit

The CSD uses two ultrasonic transducers mounted on extendable arms that can be placed on opposite outside walls of a container to actively transmit and receive an acoustic pulse though the liquid contents. The instrument simultaneously records the acoustic echo, the distance between the transducers, and the external temperature of the container under examination. An attached tablet pc is loaded with PNNL developed custom software that provides a user friendly interface. In addition, the software processes and records the collected data and calculates the velocity of sound through the liquid medium along with a measurement of the liquid's relative acoustic attenuation.

The patented CSD (US Pat \#: 7,246,522) works by launching ultrasonic pulses into a container and analyzing the pulses after they have traveled through the container and associated contents. Then, those pulses are used to accurately measure the time-of-flight and acoustic energy to compute the temperature-corrected acoustic velocity (speed of sound) and relative acoustic attenuation for characterization and classification purposes. These pulses are also used to determine if there are hidden compartments, contraband or other anomalous items hidden inside a container or bulk solid item. Additional acoustic properties (such as density, acoustic impedance, etc) may also be obtained and are currently under study at the Laboratory.

The acoustics based technology successfully utilized in the CSD is based upon years of fundamental scientific research in correlating acoustic physical property measurements - as fingerprints for classification/ discrimination of liquids and solids - using nondestructive and non-invasive means for acquiring information through a solid material or liquid-filled container.

To provide a firm engineering and scientific basis for developing the measurement capabilities and functionality of the CSD, proof-of-concept studies were conducted to test the viability and performance of 
various advanced techniques and methodologies for developing the current platform to more effectively meet the functionality and requirements of our clients. The CSD's liquid discrimination capabilities are focused on small containers, specifically over a size range of a 1" diameter test tube to that of a 10" diameter container. As a result of focusing the CSD design for examination of smaller containers, a platform was conceptualized and developed using automated distance and temperature measurement protocols, state-of-the-art broadband, piezo-composite ultrasonic transducers, advanced electronics circuit design and performance, and advanced signal processing algorithms and software that directly addresses pertinent inspection/examination issues for small containers.

In order to obtain high accuracy time-of-flight (TOF) measurements, traditional ultrasonic methods resort to the use of higher frequency transducers. However, many containers (plastic, glass) and/or fluids (castor oil, honey) exhibit high attenuation properties, which do not allow higher frequencies to penetrate effectively. This reduction in allowable frequencies reduces the TOF resolution/accuracy, which precludes the use of typical commercially available ultrasonic technologies. Therefore, research was directed toward employing an advanced pulse compression technique, whereby large amounts of ultrasonic energy are transmitted into the medium, resulting in higher signal-to-noise ratios (SNR) and more accurate TOF measurements.

Pulse compression is a technique that has been employed in both $\operatorname{RADAR}^{1,2}$ and medical ultrasound ${ }^{3,4}$. It is used to transmit large amounts of energy over a long period of time without sacrificing temporal resolution. A wide bandwidth, long duration frequency chirp is commonly used to excite the source (transmitting transducer). This pulse is received by one or more receiving transducers. Cross-correlation between the transmitted pulse and the received pulses results in a waveform containing the same time, amplitude and spectral information as the received pulse. Pulse compression has recently been used with broadband aircoupled transducers, where energy transmission, signal-to-noise ratio (SNR), and TOF accuracy are relatively low compared with conventional direct coupled ultrasound. ${ }^{5,6,7,8}$ Gan et al. ${ }^{6}$ found that pulse compression provided the air-coupled system with the ability to detect received pulses even when they were well below the noise floor due to the frequency encoded transmitted pulse. In addition, they were able to resolve closelyspaced return echoes from various reflection sources with high accuracy, which was not possible with typical ultrasonic tone burst or square wave excitation technologies. The pulse compression technique has also been used in conjunction with air-coupled ultrasound to interrogate food containers ${ }^{9}$ and detect foreign objects within food materials ${ }^{10}$. More recent work at PNNL has expanded to the use of pulse compression methods in slurries and in air-coupled applications for both material property measurements and ultrasonic imaging and flaw detection applications.

Poor SNR is very common in air-coupled ultrasonic testing due to impedance mismatches between air and most other materials. Traditional ultrasound may improve the SNR by simply using high power pulse transmission, commonly using tone burst excitation techniques. A long duration tone burst can efficiently transmit large amounts of energy into air or any other medium. However, tone burst excitation generally results in poor TOF accuracy and provides a narrow-banded response in the frequency domain. A longduration frequency sweep (chirp) can also efficiently transmit energy into a medium; however, as will be discussed later, signal processing techniques can be used to convert a long duration chirp into a compressed broadband pulse for extremely accurate TOF measurements and a correspondingly broadbanded response in the frequency domain.

Pulse compression is a signal processing technique carried out by cross-correlating a transmitted chirp with a received signal. The cross correlation function effectively locates the specific frequency pattern within the received waveform and outputs a compressed waveform containing information associated with the frequency-dependent amplitude, and transit time of the transmitted pulse. This procedure is extremely useful when trying to locate echoes within a signal whose amplitude is well below that of the noise floor. Gan et al. ${ }^{6}$ 
demonstrated an increased SNR using the pulse compression technique to locate an echo within a noisy return signal. The energy associated with the compressed cross correlation signal is directly related to the duration of the transmitted chirp pulse. Therefore, in order to achieve a higher SNR, a longer duration pulse is employed. As stated earlier, the pulse compression technique results in accurate TOF measurements. This is directly related to the frequency bandwidth of the transmitted and received pulses, where a larger bandwidth results in higher TOF resolution. Effectively, the cross correlation output will appear as a broadband pulse with a width inversely proportional to the bandwidth of the transmitted chirp. This phenomenon leads to another advantage of the pulse compression technique also known as deconvolution. For a system containing multiple echoes, a traditional ultrasonic tone burst configuration would not be able to discriminate between closely spaced echoes. However, a long-duration, broadband transmitted chirp results in a compressed cross correlation function having multiple narrow-width pulses, which allows multiple echoes to be easily resolved. Details of the measurement methodology and algorithm development have been reported by Tucker and Diaz $^{11}$.

This paper focuses on reporting the results of a recently completed series of laboratory measurements acquired with the CSD device as applied to several types of liquids (including threat liquids and precursor chemicals) that could be used in the manufacture of homemade explosives. The first phase of this internal research and development project is presented here and describes the ultrasonic measurements and discrimination trials across a subset of benign commodities and commercially available liquid products and a set of highly flammable and/or precursor liquid chemicals potentially used in the fabrication of binary liquid explosives.

\subsection{COMPARATIVE PERFORMANCE EVALUATION}

The objective of Phase 1 of this study was to further understand the critical acoustic measurement discrimination/sensitivity issues associated with quantifying the effectiveness of the CSD prototype for classifying and discriminating liquid threat materials from benign and commercially available liquids. A list of pertinent liquid threats was generated and measurements described here, were conducted in Phase 1 of this initial study to demonstrate the CSD's ability to acquire accurate and repeatable acoustic information for effectively classifying and discriminating these liquids from non-threat liquids such as alcohol-based commodities (i.e., wine, whiskey, soda, etc.). This manuscript reports on the data acquisition and preliminary results from Phase 1 of this effort, and includes a cursory evaluation for determining the sensitivity of the acoustic measurements for classification and discrimination tasks. It is envisioned that this information would found the basis for design and development of a program for creating a multi-modal (acoustic, X-ray, dielectric, etc.) commercial technology for small-container inspection at security checkpoints and airports. The focus of this effort is directed at evaluating a subset of liquid threats and common commodities.

The prototype CSD platform was used to measure the acoustic velocity and relative attenuation of 21 liquids at room temperature (approximately $70^{\circ} \mathrm{F}$ ) in Phase 1, while recording the key parameters that play a role in the accuracy and precision (repeatability) of the measurement. The CSD algorithms were designed to detect and measure discrete echoes in the container walls to compute wall thickness prior to calculating the acoustic velocity. In many cases this algorithm works well, however, more work is needed to enable a consistent and accurate wall-thickness determination under all conditions. In order to reduce the effects of container variability (wall thickness, wall material-type, container shape, contour and curvature), these measurements were conducted using the same container type (Polyethylene Terephthalate - PETE $120 \mathrm{ml}$ containers) with the exception of nitric acid which required a poly-coated glass bottle for containment. Key measurement parameters such as container diameter, container-wall temperature, acoustic velocity and relative attenuation values were recorded for each of these samples. A series of multiple trials were conducted using a set of 20 
identical PETE containers, and de-ionized, degassed water was used as the baseline liquid medium for measurement of acoustic properties. Tests were also conducted with different operators conducting measurements using the device on a subset of the initial water measurements to quantify operator variability. Finally, a set of 20 measurements were conducted on each liquid in the test set. Table 1 provides the list of liquids evaluated during Phase 1 of this research study and reported here. Phase 2 will include a rigorous statistical evaluation of all data in both Phases and in addition, will include acoustic evaluation of $150 \mathrm{ml}$ samples of 2,4,6-Trinitrotoluene in acetonitrile, Pentaerythritol Tetranitrate (PETN) in acetonitrile, Nitroglycerin in acetonitrile, RDX in acetonitrile/DMSO (90:10 ratio), and HMX in acetonitrile/DMSO (90:10 ratio); all at concentrations of $50 \mathrm{mg} / \mathrm{ml}$.

Table 1. List of 21 liquids evaluated in Phase 1 of this study.

\begin{tabular}{|c|c|c|}
\hline Liquid Name: & Concentration: & Category Descriptor: \\
\hline Acetone & $99 \%$ & Solvent/Fuel Additive \\
\hline Acetonitrile & 99.5+\% A.C.S. Reagent & Solvent \\
\hline Anhydrous Hydrazine & $98 \%$ & Ignition Source/Propellant \\
\hline Cologne (Calvin Klein ${ }^{\circledR}$ CKBe) & Not Provided & Benign Commercial Product \\
\hline De-ionized De-gassed Water $\left(\mathrm{H}_{2} \mathrm{O}\right)$ & Not Applicable & Benign Commercial Product \\
\hline Gasoline & 92 octane & Ignition Source/Fuel \\
\hline Hydrogen Peroxide $\left(\mathrm{H}_{2} \mathrm{O}_{2}\right)$ & $50 \%$ in solution & Oxidizer/Propellant/LE Component \\
\hline Irish Cream Liquor (Carolyn's ${ }^{\circledR}$ ) & $17 \%$ alcohol (34 proof) & Benign Commercial Product \\
\hline Isopropyl Alcohol & $91 \%$ concentration & Solvent \\
\hline Methanol & $99.9 \%$ & Solvent/Fuel \\
\hline Nitric Acid & $\geq 90 \%$ A.C.S. Reagent & Oxidizer/Acid/LE Component \\
\hline Nitromethane & $95+\%$ A.C.S. Reagent & Solvent/Fuel/LE Component \\
\hline Perfume $\left(\right.$ Nautica $\left.{ }^{\circledR}\right)$ & Not Provided & Benign Commercial Product \\
\hline Propylene Oxide & $99 \%$ ReagentPlus ${ }^{\circledR}$ & Thermobaric Explosive \\
\hline Red Wine (Woodbridge ${ }^{\circledR}$ Cabernet Sauvignon) & $17.5 \%$ alcohol (35 proof) & Benign Commercial Product \\
\hline Scotch $\left(\right.$ Johnnie Walker’s $\left.{ }^{\circledR}\right)$ & $40 \%$ alcohol ( 80 proof) & Benign Commercial Product \\
\hline Sulfuric Acid & 98\% A.C.S. Reagent & Acid/LE Component \\
\hline Tequila (Jose Cuervo $\left.{ }^{(}\right)$ & $40 \%$ alcohol ( 80 proof $)$ & Benign Commercial Product \\
\hline Vanilla Liquor $\left(\operatorname{Navan}^{\circledR}\right)$ & $40 \%$ alcohol ( 80 proof) & Benign Commercial Product \\
\hline Whiskey $\left(\right.$ Crown Royal $\left.{ }^{\circledR}\right)$ & $40 \%$ alcohol ( 80 proof) & Benign Commercial Product \\
\hline White Wine (Sutter Home ${ }^{\circledR}$ White Zinfandel) & $9.5 \%$ alcohol (19 proof) & Benign Commercial Product \\
\hline
\end{tabular}

Over 200 individual measurements were acquired on de-ionized, de-gassed water at near room temperature. Water was used as the liquid medium for conducting baseline measurements to evaluate and quantify container variability within the set of containers used in this study. The temperature variations noted during the measurement process were due to fluctuations resulting from the laboratory heating and air conditioning system. All measurements were recorded between 68 and $75^{\circ} \mathrm{F}$. These water trials included 10 measurements each in 20 identical PETE (plastic) containers. Once the container set was fully characterized with water, the test plan required acquisition of 20 individual acoustic measurements for each of the listed liquids in Table 1. In order to determine measurement effects due to operator variability, a subset of measurements on de-ionized de-gassed water were again conducted using a different operator on the CSD system. In addition, 40 measurements of de-ionized de-gassed water were taken with no re-coupling of the transducers between measurements. The CSD platform was locked onto the container and the measurement button was pressed 40 times to evaluate variation in the measurement results attributed to the device only. Finally, a set of physical container diameter measurements were taken on each of the 20 PETE containers using a digital caliper, and these data were recorded and analyzed to provide information associated with the true state variation in container dimensions and correlation with the CSD measured container diameters. In 
all, nearly 700 individual measurement trials were conducted during Phase 1 of this study. A preliminary statistical evaluation was conducted on these data and a more rigorous analysis will be reported at a later date once the TNT, PETN, RDX, HMX and Nitroglycerin samples have been measured. This is expected to occur in mid-summer, 2008.

\subsection{PHASE 1 PERFORMANCE TESTING RESULTS}

The Phase 1 effort described here was based primarily upon three datasets. The first involved positioning a single bottle of de-ionized water in the CSD platform and taking 40 consecutive readings for distance, velocity, attenuation, and temperature without re-positioning or re-coupling the container. For the second dataset, distance, velocity, attenuation, and temperature measurements were generated using 20 "identical" bottles containing de-ionized, de-gassed water. For these observations, the bottles were re-positioned and recoupled within the CSD platform prior to each individual measurement. The third dataset involved collecting distance, velocity, attenuation, and temperature measurements using the same type of bottle used for the other two datasets, but with a variety of liquids.

The main objective of the statistical analysis of the data was to investigate the potential of using the CSD to distinguish/discriminate between liquids of concern (LE's and their precursors) and liquid commodities that are consumable and/or benign to transportation safety (liquor, water, etc.). Statistical methods were explored that would allow identification, or classification, of a liquid contained in a bottle given corresponding measurements of distance, velocity, attenuation, and temperature. Confidence levels associated with the classifications were also generated. These statistical methods also involved quantifying the uncertainty associated with the distance, velocity, attenuation, and temperature measurements, as well as the uncertainty due to different operators of the measurement apparatus. Table 2 provides a summary of the averaged data from the three (3) primary data sets analyzed in the initial Phase 1 study along with the standard deviations.

Table 2. Summary of Phase 1 data.

\begin{tabular}{|c|c|c|c|c|c|}
\hline Liquid & $\begin{array}{l}\text { Ave. } \\
\text { Velocity } \\
\text { (m/s) }\end{array}$ & $\begin{array}{c}\text { Velocity } \\
\text { Std Dev } \\
(\mathrm{m} / \mathrm{s})\end{array}$ & $\begin{array}{c}\text { Relative } \\
\text { Attenuation } \\
\text { (unitless) }\end{array}$ & $\begin{array}{c}\text { Relative } \\
\text { Attenuation } \\
\text { Std. Dev }\end{array}$ & $\begin{array}{c}\text { Number } \\
\text { of } \\
\text { Trials } \\
\end{array}$ \\
\hline Acetone & 1172.781 & 5.136 & 0.9565 & 0.0792 & 20 \\
\hline Acetonitrile & 1275.916 & 0.682 & 0.6712 & 0.0438 & 20 \\
\hline Methanol & 1108.681 & 7.691 & 0.6745 & 0.0648 & 20 \\
\hline Perfume (Nautica ${ }^{\circledR}$ ) & 1263.172 & 7.101 & 0.6554 & 0.0786 & 20 \\
\hline Cologne (Calvin Klein ${ }^{\circledR}$ CKBe) & 1286.350 & 7.138 & 0.6348 & 0.0548 & 20 \\
\hline Gasoline (92 Octane) & 1149.085 & 0.487 & 0.6783 & 0.0399 & 20 \\
\hline Hydrogen Peroxide ( $50 \%$ in solution) & 1625.768 & 2.762 & 0.6747 & 0.0447 & 20 \\
\hline Isopropyl Alcohol (91\%) & 1214.029 & 1.119 & 0.8789 & 0.0943 & 20 \\
\hline Sulfuric Acid & 1359.412 & 1.043 & 0.6357 & 0.0730 & 20 \\
\hline Nitric Acid & 1342.502 & 6.610 & 0.6079 & 0.0927 & 20 \\
\hline Propylene Oxide & 1170.968 & 0.525 & 0.7875 & 0.0832 & 20 \\
\hline Anhydrous Hydrazine & 2067.550 & 1.379 & 0.6656 & 0.0621 & 20 \\
\hline Nitromethane & 1337.749 & 0.400 & 0.6847 & 0.0221 & 20 \\
\hline $\begin{array}{l}\text { De-ionized, de-gassed water (10 trials each in } \\
20 \text { identical PETE containers, for } 200 \text { total } \\
\text { measurements) }\end{array}$ & 1478.322 & 6.841 & 0.6389 & 0.0542 & 200 \\
\hline Irish Cream Liquor (Carolyn's ${ }^{\circledR} 17 \%$ alcohol) & 1588.879 & 3.635 & 1.3120 & 0.1709 & 20 \\
\hline Vanilla Liquor (Navan ${ }^{\circledR} 40 \%$ alcohol) & 1586.823 & 4.098 & 0.7272 & 0.0701 & 20 \\
\hline Scotch (Johnnie Walker's ${ }^{\circledR} 40 \%$ alcohol) & 1580.185 & 4.575 & 0.7319 & 0.052 & 20 \\
\hline
\end{tabular}




\begin{tabular}{|c|c|c|c|c|c|}
\hline Whiskey (Crown Royal $^{\circledR} 40 \%$ alcohol) & 1580.916 & 1.13 & 0.7281 & 0.0529 & 20 \\
\hline Tequila (Jose Cuervo ${ }^{\circledR} 40 \%$ alcohol) & 1583.601 & 0.592 & 0.618 & 0.0242 & 20 \\
\hline $\begin{array}{l}\text { Red Wine (Woodbridge }{ }^{\circledR} \text {, Cabernet Sauvignon } \\
17.5 \% \text { alcohol) }\end{array}$ & 1566.915 & 2.71 & 0.7119 & 0.0778 & 20 \\
\hline $\begin{array}{l}\text { White Wine (Sutter Home }{ }^{\circledR} \text {, White Zinfandel } \\
9.5 \% \text { alcohol) }\end{array}$ & 1555.280 & 6.382 & 0.6408 & 0.0366 & 20 \\
\hline $\begin{array}{l}\text { De-ionized, de-gassed water (no-decoupling } \\
\text { between individual measurements) }\end{array}$ & 1482.413 & 0.3675 & 0.6197 & 0.0059 & 40 \\
\hline
\end{tabular}

A scatterplot matrix analysis of pairwise plots comparing relative attenuation, acoustic velocity, and temperature values for the 40 observations of de-ionized, de-gassed water with no de-coupling between measurements was conducted. This analysis did not suggest any strong correlations among these three variables. The correlation values shown below in Table 3 re-enforce the absence of strong correlations.

Table 3. Correlation values from pairwise analyses of the first dataset.

\begin{tabular}{|c|c|c|c|}
\hline Parameter & Attenuation & Velocity & Temperature \\
\hline Attenuation & 1.000 & 0.299 & 0.078 \\
\hline Velocity & 0.299 & 1.000 & 0.432 \\
\hline Temperature & 0.078 & 0.432 & 1.000 \\
\hline
\end{tabular}

The relative standard deviations (RSDs) for attenuation, velocity, and temperature were all less than $1 \%$. Relative standard deviation is defined as the standard deviation calculated using the measured values for a particular variable divided by the mean of those values multiplied by 100 . This indicates that the measurement process is very consistent.

$$
\begin{aligned}
& \operatorname{RSD}(\text { Attenuation })=0.955 \\
& \operatorname{RSD}(\text { Velocity })=0.025 \\
& \operatorname{RSD}(\text { Temperature })=0.208
\end{aligned}
$$

The second dataset included 10 observations for each of 20 bottles containing de-ionized, degassed water, where two of the bottles in this set of containers provided an additional 10 observations each. For these observations (measurements), the bottles were re-positioned in the apparatus prior to each reading. Thus, there were some differences in distance readings even for a given bottle. An analysis of the distance measurements and their distribution for the 20 bottles was conducted. A pooled standard deviation for distance, calculated over the set of 20 bottles, was 0.0073 inches. Once again, a pairwise analyses was conducted for attenuation, velocity, distance, and temperature as collected from the 20 bottles containing deionized, degassed water. The resultant analysis indicated strong correlation between the temperature values for the CSD platform and those taken using a commercially available laser gun temperature measurement platform, as expected. This analysis also indicated that aside from the temperature values, there did not appear to be strong correlations among the other variables from the water data. The water data did have two clusters of data points centered around $1473 \mathrm{~m} / \mathrm{s}$ and $1487 \mathrm{~m} / \mathrm{s}$ respectively, and this is due to an issue with the detection algorithm of the CSD where consistent capture of the appropriate echo within a received echo packet is sometimes incorrect. This issue will be resolved in the next version of the detection algorithm software.

For the third dataset, measurements for distance (bottle diameter), temperature (from both CSD and the gun), attenuation, and velocity were collected on 21 different liquids, with 20 observations each, all in the same container, and at near room-temperature. However, for the water trials, 30 observations were taken (with a 
different operator for the CSD measurement apparatus), plus the 200 observations from the second dataset were included in this analysis, yielding 230 observations for de-ionized, de-gassed water. A summary of the averaged results from the third dataset are provided visually in Figure 2.

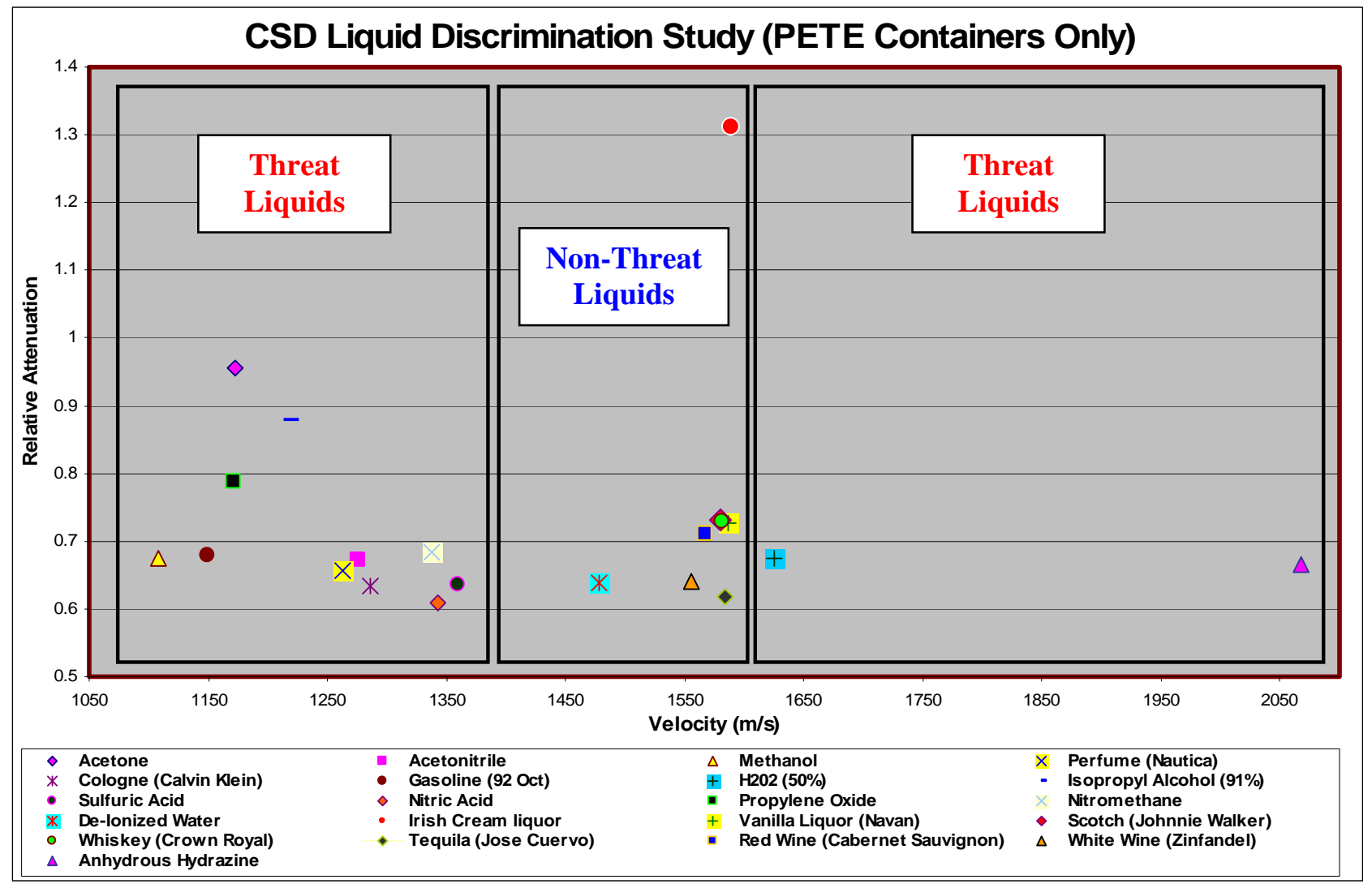

Figure 2. Summary plot depicting averaged data from the third dataset. Black-outlined boxes indicate groupings for separation/discrimination of the liquids evaluated in this Phase 1 effort.

Figure 2 shows the mean attenuation versus the mean velocity for each of the 21 liquids. A preliminary statistical analysis of these data indicates that some liquids are well separated from the others and should be fairly easy to identify using statistical classification methods. However, certain liquids such as scotch, whiskey, and vanilla liquor have very similar combinations of attenuation and velocity. Such liquids could be more challenging to classify and discriminate precisely. However, for applications in transportation and airline security, it would be most beneficial to have such items as consumable drinks and liquors grouped as "similar" from an acoustic measurements perspective. There is definitely a greater interest in distinguishing between broader categories of liquids, particularly those posing potential hazards. An analysis of the distributions of temperature, both from CSD and the gun, attenuation, and velocity for all available observations taken for the 21 liquids was conducted as well. The measured container distances were all quite consistent, and the only feature of interest brought out in the distance analysis was that measurements on nitric acid were taken using a larger bottle (approximately 3.5 in.) compared to the bottles used for the other liquids (approximately 1.9 inches in diameter).

Two basic classification algorithms were used to investigate the ability to distinguish between the different liquids considered in the third dataset. These methods were the classification and regression tree algorithm 
(CART), and the linear discrimination algorithm (LDA). Results from these two methods presented here represent preliminary runs using the commercially available versions of these methods available in the software package R. In both cases, "Liquid" was the response variable to be predicted based on the independent variables "Velocity", "Attenuation", and "Temperature". No adjustments (standardization, averaging, etc.) were made to the independent variables for these initial runs. As expected, certain liquids are easily classified/identified using these two statistical methods. Other liquids are more difficult to classify/identify correctly. More sophisticated classification algorithms will be considered in Phase 2, with the expectation that they should be more effective at discriminating between the liquids evaluated here. A classification "tree" resulting from using the CART algorithm on all of the available data was generated, and nearly all of the branching that occurs on this tree is based upon velocity, and only a few branches are based upon attenuation. No branches were based upon temperature. The CART model incorporates a "smearing" affect where probabilities of association are assigned to each liquid category as each "new" observation (measurement) is tested. For example, given a certain combination of measured velocity, attenuation, and temperature values obtained for a certain liquid, the CART model assigns probabilities of association to the different liquids such as

$\begin{array}{lc}\text { Acetone } & 0.0000000 \\ \text { Acetonitrile } & 0.0000000 \\ \text { Anhydrous Hydrazine } & 0.0000000 \\ \text { CKBe Cologne } & 0.0000000 \\ \text { De-ionized water } & 0.0000000 \\ \text { Gasoline } & 0.0000000 \\ \text { H2O2 50\% } & 0.0000000 \\ \text { Irish Cream } & 0.0000000 \\ \text { Isopropyl Alc (91\%) } & 0.0000000 \\ \text { Methanol } & 0.0000000 \\ \text { Nautica } & 0.0000000 \\ \text { Nitric Acid } & 0.0000000 \\ \text { Nitro Methane } & 0.0000000 \\ \text { Propylene Oxide } & 0.0000000 \\ \text { Red Wine } & 0.0000000 \\ \text { Scotch } & 0.7272727 \\ \text { Sulfuric Acid } & 0.0000000 \\ \text { Tequila } & 0.0000000 \\ \text { Vanilla Liquor } & 0.1818182 \\ \text { Whiskey } & 0.0909091 \\ \text { White Wine } & 0.0000000\end{array}$

For this example, the liquid would be classified as scotch because it has the highest associated probability. A confusion matrix was generated using a "leave-one-out" re-sampling approach. The process involves repeatedly selecting a random observation (velocity, attenuation, and temperature measurements) from among the observations available for a given liquid, and applying the CART model fit using the remaining observations (the remaining observations over all liquids) to the selected observation. The probabilities of association are summed over the repeated tests for a given liquid, and the results are divided by the number of tests for that liquid.

As with the CART analysis, LDA also assigns probabilities of association to each liquid category for each "new" observation tested. The same "leave-one-out" re-sampling approach used for CART was employed to generate a confusion matrix based on the LDA classification method. Table 4 provides the results from the 
confusion matrix generated from the LDA process and lists the actual confusion matrix values for a set of over 10,000 trials using the LDA model and observations from the Phase 1 data.

\begin{tabular}{|c|c|c|c|c|c|c|c|c|c|c|c|c|c|c|c|c|c|c|c|c|c|}
\hline & 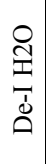 & 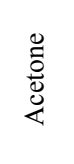 & 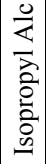 & 尽 & 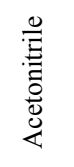 & 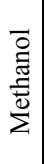 & 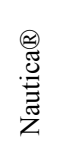 & 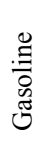 & 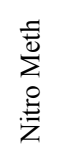 & 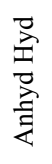 & 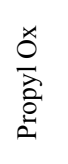 & 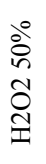 & 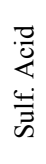 & $\frac{\mathscr{J}}{\stackrel{\mathscr{d}}{\simeq}}$ & 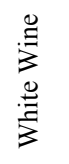 & 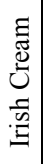 & 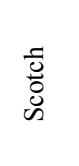 & 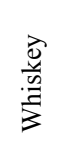 & 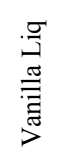 & 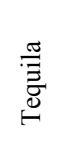 & 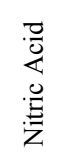 \\
\hline De-I H2O & 1 & 0 & 0 & 0 & 0 & 0 & 0 & 0 & 0 & 0 & 0 & 0 & 0 & 0 & 0 & 0 & 0 & 0 & 0 & 0 & 0 \\
\hline Acetone & 0 & 0.99 & 0 & 0 & 0 & 0 & 0 & 0 & 0 & 0 & 0.01 & 0 & 0 & 0 & 0 & 0 & 0 & 0 & 0 & 0 & 0 \\
\hline Iso. Alc. & 0 & 0 & 1 & 0 & 0 & 0 & 0 & 0 & 0 & 0 & 0 & 0 & 0 & 0 & 0 & 0 & 0 & 0 & 0 & 0 & 0 \\
\hline $\mathrm{CKBe}^{\circledR}$ & 0 & 0 & 0 & 1 & 0 & 0 & 0 & 0 & 0 & 0 & 0 & 0 & 0 & 0 & 0 & 0 & 0 & 0 & 0 & 0 & 0 \\
\hline Acetonitrile & 0 & 0 & 0 & 0 & 0.96 & 0 & 0.04 & 0 & 0 & 0 & 0 & 0 & 0 & 0 & 0 & 0 & 0 & 0 & 0 & 0 & 0 \\
\hline Methanol & 0 & 0 & 0 & 0 & 0 & 1 & 0 & 0 & 0 & 0 & 0 & 0 & 0 & 0 & 0 & 0 & 0 & 0 & 0 & 0 & 0 \\
\hline Nautica $\mathbb{R}$ & 0 & 0 & 0 & 0 & 0.24 & 0 & 0.76 & 0 & 0 & 0 & 0 & 0 & 0 & 0 & 0 & 0 & 0 & 0 & 0 & 0 & 0 \\
\hline Gasoline & 0 & 0 & 0 & 0 & 0 & 0 & 0 & 1 & 0 & 0 & 0 & 0 & 0 & 0 & 0 & 0 & 0 & 0 & 0 & 0 & 0 \\
\hline Nitro Meth & 0 & 0 & 0 & 0 & 0 & 0 & 0 & 0 & 0.9 & 0 & 0 & 0 & 0 & 0 & 0 & 0 & 0 & 0 & 0 & 0 & 0.1 \\
\hline Anhyd Hyd & 0 & 0 & 0 & 0 & 0 & 0 & 0 & 0 & 0 & 1 & 0 & 0 & 0 & 0 & 0 & 0 & 0 & 0 & 0 & 0 & 0 \\
\hline Propyl Ox & 0 & 0.04 & 0 & 0 & 0 & 0 & 0 & 0 & 0 & 0 & 0.96 & 0 & 0 & 0 & 0 & 0 & 0 & 0 & 0 & 0 & 0 \\
\hline $\mathrm{H} 2 \mathrm{O} 250 \%$ & 0 & 0 & 0 & 0 & 0 & 0 & 0 & 0 & 0 & 0 & 0 & 1 & 0 & 0 & 0 & 0 & 0 & 0 & 0 & 0 & 0 \\
\hline Sulf.Acid & 0 & 0 & 0 & 0 & 0 & 0 & 0 & 0 & 0 & 0 & 0 & 0 & 1 & 0 & 0 & 0 & 0 & 0 & 0 & 0 & 0 \\
\hline Red Wine & 0 & 0 & 0 & 0 & 0 & 0 & 0 & 0 & 0 & 0 & 0 & 0 & 0 & 0.85 & 0.08 & 0 & 0.03 & 0.02 & 0 & 0.03 & 0 \\
\hline White Wine & 0 & 0 & 0 & 0 & 0 & 0 & 0 & 0 & 0 & 0 & 0 & 0 & 0 & 0.2 & 0.79 & 0 & 0 & 0 & 0 & 0 & 0 \\
\hline Irish Cream & 0 & 0 & 0 & 0 & 0 & 0 & 0 & 0 & 0 & 0 & 0 & 0 & 0 & 0 & 0 & 1 & 0 & 0 & 0 & 0 & 0 \\
\hline Scotch & 0 & 0 & 0 & 0 & 0 & 0 & 0 & 0 & 0 & 0 & 0 & 0 & 0 & 0.08 & 0 & 0 & 0.39 & 0.29 & 0.11 & 0.12 & 0 \\
\hline Whiskey & 0 & 0 & 0 & 0 & 0 & 0 & 0 & 0 & 0 & 0 & 0 & 0 & 0 & 0 & 0 & 0 & 0.29 & 0.38 & 0.19 & 0.14 & 0 \\
\hline Vanilla Liq & 0 & 0 & 0 & 0 & 0 & 0 & 0 & 0 & 0 & 0 & 0 & 0 & 0 & 0 & 0 & 0 & 0.12 & 0.25 & 0.51 & 0.13 & 0 \\
\hline Tequila & 0 & 0 & 0 & 0 & 0 & 0 & 0 & 0 & 0 & 0 & 0 & 0 & 0 & 0 & 0 & 0 & 0.11 & 0.15 & 0.1 & 0.65 & 0 \\
\hline Nitric Acid & 0 & 0 & 0 & 0 & 0 & 0 & 0 & 0 & 0.22 & 0 & 0 & 0 & 0 & 0 & 0 & 0 & 0 & 0 & 0 & 0 & 0.78 \\
\hline
\end{tabular}

Table 4. Resultant confusion matrix values for a set of over 10,000 trials using the LDA model and observations from the Phase 1 data.

\subsection{DISCUSSIONS, CONCLUSIONS AND FUTURE WORK}

From the work conducted to date at PNNL, tests have demonstrated the ability of the CSD technology to rapidly and effectively classify and discriminate several types of liquids (including threat liquids and precursor chemicals) in PETE containers with the necessary accuracy and reliability, and with the demonstrated ability to consistently "group" and separate many consumable/benign commercial products from LE precursor chemicals. In Phase 1 of this effort a subset of liquids was evaluated and a preliminary statistical analysis of the data was conducted. In Phase 2 of this study, more measurements will be conducted on samples of TNT, PETN, Nitroglycerin, RDX, and HMX; all at concentrations of $50 \mathrm{mg} / \mathrm{ml}$. In addition, a more rigorous statistical evaluation will be conducted, to include more advanced statistically-based discrimination algorithms for use on these data to more effectively discriminate dangerous LE's and their precursors from commercially available consumable liquids and other benign liquid products. From the data evaluated in this study, the CSD demonstrated the ability of using acoustic property measurements to classify and/or discriminate threat liquids from non-threat liquids. The only liquids that were not considered threatliquids but were classified acoustically as threat liquids were the cologne and perfume samples evaluated here. These liquids were similar to acetonitrile and isopropyl alcohol from an acoustic perspective.

As the set of measured liquids grows, the capability for acoustic classification and discrimination of liquids will be better quantified. Also, as additional measurement properties (signatures) are obtained, such as density, acoustic impedance, etc., the ability to employ more advanced mathematical methods for 
discrimination will be studied. More work is needed to optimize the CSD's algorithms to better address the effects of container variations on the acoustic property measurements.

Fluid characterization testing with the CSD also reinforced what the primary factors are that influence the accuracy and repeatability of attenuation measurements. Factors include transducer coupling to the container, beam divergence, container material, container geometry, distance measurement, temperature measurement, transducer alignment, and frequency bandwidth of the transducers. The most crucial component for accurate attenuation measurements is coupling. In order to efficiently transmit ultrasonic energy into a container, adequate coupling is required. While traditionally, an ultrasonic gel couplant is used to achieve coupling, tests at PNNL have demonstrated the viability of utilizing a custom-designed dry couplant membrane that eliminates the utilization of gel or other coupling agents. Container wall material plays a significant role, in that different materials will absorb varying amounts of ultrasonic energy. In addition, container material and geometry variances lead to variations in reflection coefficients, thereby changing the amount of energy transmitted and reflected at each interface. Container walls can also be flexible leading to incorrect distance measurements. This is overcome by the design of the CSD container fixture which is specifically designed to accurately measure the distance between transducer faces in real time. Transducer alignment is critical for the accurate measure of attenuation. The transducer faces must be directly opposite and aligned parallel to each other for accurate transmission and reception of ultrasonic waves. The CSD fixture has been machined with strict tolerances to allow for precise transducer alignment but future work will focus on alternate and more sophisticated approaches to the distance measurement process. As with velocity, distance and temperature measurements are critical for accurate attenuations measurements. Divergence is a physical phenomenon associated with ultrasonic wave propagation whereby the energy begins to "spread out" as a function of distance from the source, similar to a flashlight. The divergence error varies depending on the ultrasonic wave speed in the fluid and the area of the transducer face that is effectively coupled to the container wall. This divergence of the beam spreads the energy over a larger area, which decreases the energy along the straight-line path between the transducers. Since divergence is dependent upon the fluid velocity and container geometry, further refinement of the CSD's computer algorithms is planned, to compensate for this "apparent" change in energy. Finally, transducer bandwidth plays a role in accurate attenuation measurements. With a larger frequency range over which to calculate the attenuation spectra, a more consistent estimate of the relative attenuation can be obtained.

With regard to acoustic velocity, more work is slated for addressing real-time wall thickness compensation from the detection and processing of wall echoes in the processed data. Also, consistent detection of the appropriate echoes in the received wave "packets" from the processed waveforms will be addressed in future work. This issue was prevalent in the water data from the second dataset described here.

\section{REFERENCES}

1. Axelsson, S.R.J., 2004. Noise radar using random phase and frequency modulation. IEEE Transactions on Geoscience and Remote Sensing 42(11):2370-2384.

2 Rajeswari, K.R., N. Gangatharan, G.E.M. Abraham, G.S.V.R.K. Rao, and D.E. Rani. 2003. Algorithm for design of pulse compression radar codes. Electronics Letters 39(11):865-867.

3 Behar, V. and D. Adam. 2004. Parameter optimization of pulse compression in ultrasound imaging systems with coded excitation. Ultrasonics 42(10):1101-1109.

4. Chang, Y.F. 2003. A software controlled pulse compression technique applied to ultrasonic nondestructive testing. Journal of the Chinese Institute of Engineers, 26(2):147-153.

5. Berriman, J., P. Purnell, D.A. Hutchins, and A. Neild. 2005. Humidity and aggregate content correction factors for air-coupled ultrasonic evaluation of concrete. Ultrasonic 43(4):211-217. 
6. Gan, T.H., D.A. Hutchins, D.R. Billson, and D.W. Schindel. 2001. The use of broadband acoustic transducers and pulse-compression techniques for air-coupled ultrasonic imaging. Ultrasonics 39(3): 181-194.

7 Gan T.H., D.A. Hutchins, D.R. Billson, and F.C. Wong. 2001. Ultrasonic tomographic imaging of an encased, highly attenuating solid medium. Research in Nondestructive Evaluation 13 (3):131-152.

8 Gan, T.H., D.A. Hutchins, and R.J. Green. 2004. A swept frequency multiplication technique for air-coupled ultrasonic NDE. IEEE Transactions on Ultrasonics, Ferroelectrics, and Frequency Control, 51(10):1271-1279.

9 Gan, T.H., D.A. Hutchins, and D.R. Billson. 2002. Preliminary studies of a novel air-coupled ultrasonic inspection system for food containers. Journal of Food Engineering, 53(4):315-322.

10 Cho, B.K. and J.M.K. Irudayaraj. 2003. Foreign object and internal disorder detection in food materials using noncontact ultrasound imaging. Journal of Food Science, 68(3):967-974.

11. Tucker, B.J., A.A. Diaz, 2005. Advanced ultrasonic measurement methodology for non-invasive interrogation and identification of fluids in sealed containers. Nondestructive Detection and Measurement for Homeland Security III Conference, SPIE Conference Paper 5769-06, March 6-9, 2005, San Diego, CA. 